\title{
ESTIMATIVA DA SUSCEPTIBILIDADE À COMPACTAÇÃO E DO SUPORTE DE CARGA DO SOLO COM BASE EM PROPRIEDADES FÍSICAS DE SOLOS DO RIO GRANDE DO SUL ${ }^{(1)}$
}

\author{
Luis Eduardo Akiyoshi Sanches Suzuki ${ }^{(2)}$, Dalvan José Reinert ${ }^{(3)}$, \\ José Miguel Reichert $^{(3)}$ \& Cláudia Liane Rodrigues de Lima ${ }^{(4)}$
}

\begin{abstract}
RESUMO
O conhecimento das relações entre propriedades físicas e mecânicas do solo pode contribuir no desenvolvimento de funções de pedotransferência, que permitam estimar outras propriedades do solo de difícil mensuração. Neste trabalho, objetivou-se avaliar a relação entre a susceptibilidade à compactação e o suporte de carga com propriedades físicas de solos do sul do Brasil. Foram avaliadas a resistência à penetração, a umidade, a densidade e a compressibilidade de seis solos. A resistência à penetração pode ser estimada pelo modelo que considera a umidade e densidade do solo. Solos com maior densidade inicial apresentaram menor susceptibilidade à compactação e menor deformação, quando submetidos a pressões externas. Quanto maior a resistência do solo à penetração, menor é a deformação e maior é a capacidade de suporte de carga, embora isso não indique solos com qualidade física adequada para as culturas; quanto maior a deformação do solo, maior a susceptibilidade à compactação e menor a capacidade de suporte de carga. A susceptibilidade de um solo à compactação e sua capacidade de suporte de carga podem ser estimadas, respectivamente, pela densidade inicial e pela resistência do solo à penetração.
\end{abstract}

Termos de indexação: funções de pedotransferência, índice de compressão, pressão de preconsolidação, densidade do solo, resistência à penetração.

\footnotetext{
(1) Parte da Tese de Mestrado do primeiro autor. Trabalho apresentado na XVI Reunião Brasileira de Manejo e Conservação do Solo e da Água, Aracaju (SE), 2006. Recebido para publicação em maio de 2007 e aprovado em março de 2008.

(2) Professor da Universidade Estadual do Rio Grande do Sul - UERGS. Rua Dr. José Bisognin 250, Bairro São Cristovão, CEP 99700-000 Erechim (RS). E-mail: dusuzuki@gmail.com

(3) Professor do Departamento de Solos, Universidade Federal de Santa Maria - UFSM. Av. Roraima 1000, CEP 97105-900 Santa Maria (RS). Pesquisador do CNPq. E-mail: dalvan@smail.ufsm.br; reichert@smail.ufsm.br.

(4) Pesquisadora da Embrapa Clima Temperado, Rodovia BR 392, km 78, Caixa Postal 403, CEP 96001-970 Pelotas (RS). E-mail: clrlima@yahoo.com.br
} 


\title{
SUMMARY: ESTIMATING SOIL SUSCEPTIBILITY TO COMPACTIONAND LOAD SUPPORT CAPACITY BASED ON PHYSICAL PARAMETERS OF SOILS FROM RIO GRANDE DO SUL STATE
}

\begin{abstract}
Quantifying the relationship between physical and mechanical soil properties can contribute to the development of pedotransfer functions that allow estimating hard-tomeasure soil properties. The objective of this study was to evaluate the interrelations between susceptibility to compaction and load support with some physical properties of soils from Southern Brazil. Penetration resistance, moisture, bulk density and compressibility of six soils were evaluated. In a model including soil moisture and bulk density as independent variables, the relation with penetration resistance values obtained in the field was high. Soils with higher initial bulk density were less susceptible to compaction and exhibited less deformation under external loads. With increasing soil penetration resistance, less deformation and greater load support were observed, which does not necessarily indicate a satisfactory soil physical quality for crop cultivation. The greater the soil deformation, the higher is the susceptibility to compaction and the lower is its load support. The compaction susceptibility and load support of a soil can be estimated, respectively, by its initial bulk density and resistance to penetration.
\end{abstract}

Index terms: pedotransfer functions, compression index, precompression stress, bulk density, soil resistance to penetration.

\section{INTRODUÇÃO}

A estimativa do comportamento mecânico do solo a partir de propriedades ou atributos de fácil obtenção, como a densidade e a resistência à penetração, apresenta dificuldades, visto que essas últimas dependem de fatores relacionados com as propriedades ou atributos de cada solo. De acordo com Larson et al. (1980), essa dificuldade está relacionada com a complexidade, variabilidade e forças naturais aplicadas aos solos. Smith et al. (1997b) apontam que a compressibilidade e a compactabilidade não apresentam clara relação entre si. Portanto, o desenvolvimento de funções que facilitem a compreensão da susceptibilidade do solo à compactação depende do efeito da compactação nas propriedades físicas do solo.

O estudo das relações entre as propriedades físicas e mecânicas do solo pode contribuir para o entendimento e desenvolvimento de funções de pedotransferência. Trabalhos nesse sentido vêm sendo realizados tanto no exterior (Alexandrou \& Earl, 1998; Canarache et al., 2000; Mosaddeghi et al., 2003; Horn \& Fleige, 2003) como no Brasil (Imhoff et al., 2004; Dias Júnior et al., 2004; Lima et al., 2006), mas ainda há necessidade de informações para o desenvolvimento de melhores funções ou para outras condições de clima e solo.

De acordo com Alexandrou \& Earl (1998), a estimativa da pressão de preconsolidação por meio de propriedades do solo de fácil obtenção fornece medida útil para o planejamento e manejo de sistemas agrícolas. Dias Júnior et al. (2004) relatam que o método para a determinação da pressão de preconsolidação está difundido, porém são necessários métodos alternativos que possam ser utilizados no campo. Uma alternativa seria a calibração de seus valores com outras medidas de fácil e rápida obtenção em campo.

A pressão de preconsolidação, que permite verificar a estabilidade mecânica de solos, pode ser estimada por meio de funções de pedotransferência, considerando a textura, a agregação e a umidade do solo (Horn \& Fleige, 2003). Dias Júnior et al. (2004) e Lima et al. (2006) verificaram relação entre a resistência à penetração e a pressão de preconsolidação. O uso de equações de pedotransferência seria não apenas simplificação do método, mas também economia de tempo na determinação da pressão de preconsolidação, podendo ser utilizada como critério auxiliar de decisão sobre o momento adequado para realizar as operações mecanizadas (Dias Júnior et al., 2004).

Poucos têm sido os trabalhos relativos a funções de pedotransferência, principalmente para o sul do Brasil. Além disso, quais propriedades físicas relacionam-se com as mecânicas e em que situações essas relações ocorrem são questões ainda não respondidas plenamente. A obtenção dessas relações, além de possibilitar a criação de funções de pedotransferência, pode facilitar o entendimento do comportamento mecânico do solo, visto que a avaliação das propriedades físicas dos distintos solos está mais avançada. A criação de funções sob diferentes condições, tanto de solos e clima, pode contribuir no entendimento do comportamento do solo, em termos de mecânica.

Neste trabalho, objetivou-se avaliar a relação entre a susceptibilidade à compactação e o suporte de carga com propriedades físicas de seis solos do sul do Brasil. 


\section{MATERIAL E MÉTODOS}

Foram estudados seis solos do Rio Grande do Sul, classificados segundo Santos et al. (2006): Argissolo Vermelho distrófico arênico (PVd2), Argissolo Vermelho distrófico latossólico (PVd3), Argissolo Vermelho-Amarelo alumínico típico (PVAa), Latossolo Vermelho distrófico típico (LVd), Latossolo Vermelho aluminoférrico típico (LVaf) e Latossolo Vermelho distroférrico típico (LVdf). Estes solos enquadram-se, respectivamente, nas unidades de mapeamento (UM) (Brasil, 1973; Streck et al., 2002): São Pedro, Alto das Canas, Júlio de Castilhos, Passo Fundo, Erechim e Santo Ângelo. As UM São Pedro e Alto das Canas localizam-se na região da Depressão Central, sendo a primeira tendo como material de origem arenitos e a segunda folhelhos. A UM Júlio de Castilhos localizase na borda do Planalto Médio com a Depressão Central, enquanto as UM Passo Fundo e Santo Ângelo situam-se no Planalto Médio e a UM Erechim no Alto Uruguai, sendo todas estas UM formadas por basalto e, para a UM Passo Fundo, uma mistura de arenito com basalto.

Os solos foram amostrados no primeiro semestre de 2004 e 2005, em propriedades agrícolas, em parcelas experimentais, com 31 distintas condições de manejo (10 sistemas de manejo para o PVd2, quatro sistemas de manejo para os solos PVd3, PVAa, LVd, seis sistemas de manejo para o LVaf e três sistemas de manejo para o LVdf), predominando o sistema plantio direto. No período da coleta, nas áreas, encontravamse as culturas da soja e, apenas em quatro manejos no PVd2, a cultura do feijão.

A análise granulométrica foi realizada pelo método da pipeta (Embrapa, 1997), com três repetições em laboratório. A dispersão das amostras de solo foi por meio de agitação horizontal com $120 \mathrm{rpm}$ durante $4 \mathrm{~h}$ e utilizando $\mathrm{NaOH} 6 \%$ (dispersante químico) e duas esferas de nylon (dispersante mecânico), conforme método descrito por Suzuki et al. (2004a,b) (Quadro 1).

Para o teste de compressão do solo, foram coletadas amostras com estrutura de solo preservada, em cilindros com altura de $0,025 \mathrm{~m}$ e diâmetro de $0,061 \mathrm{~m}$, na camada de 0,08-0,13 m, onde freqüentemente é relatada a presença de maior estado de compactação no sistema plantio direto (Silva, 2003; Genro Júnior et al., 2004; Suzuki, 2005), decorrente do acúmulo de pressões pelo tráfego de máquinas. Depois de preparadas, as amostras foram saturadas por capilaridade e, em seguida, equilibradas à tensão de $33 \mathrm{kPa}$, utilizando câmaras de pressão de Richards (Klute, 1986). Em seguida, foram submetidas ao teste de compressão uniaxial, com aplicação de cargas sucessivas e estáticas de 12,$5 ; 25 ; 50 ; 100 ; 200 ; 400$; 800 e $1.600 \mathrm{kPa}$, no consolidômetro de modelo S-450 Terraload (Durham Geo- Interprises) com aplicação de pressão por meio de ar comprimido. Cada carga foi aplicada por um tempo de cinco minutos, pois, de

Quadro 1. Análise granulométrica nas camadas de 0,05-0,10 e 0,10-0,15 m para três Argissolos e três Latossolos em estudo

\begin{tabular}{|c|c|c|c|c|c|}
\hline \multirow{2}{*}{ Camada } & \multicolumn{3}{|c|}{ Areia } & \multirow{2}{*}{ Silte } & \multirow{2}{*}{ Argila } \\
\hline & Total & Grossa & Fina & & \\
\hline \multirow[t]{2}{*}{$\mathrm{m}$} & & + & $g^{-1}$ & 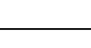 & - \\
\hline & \multicolumn{5}{|c|}{ Argissolo Vermelho distrófico arênico (PVd2) } \\
\hline $0,05-0,10$ & 660 & 196 & 464 & 237 & 103 \\
\hline \multirow[t]{2}{*}{$0,10-0,15$} & 655 & 187 & 468 & 253 & 92 \\
\hline & \multicolumn{5}{|c|}{ Argissolo Vermelho distrófico latossólico (PVd3) } \\
\hline $0,05-0,10$ & 408 & 246 & 162 & 325 & 267 \\
\hline \multirow[t]{2}{*}{$0,10-0,15$} & 399 & 245 & 155 & 342 & 259 \\
\hline & \multicolumn{5}{|c|}{ Argissolo Vermelho-Amarelo alumínico típico (PVAa) } \\
\hline $0,05-0,10$ & 149 & 54 & 95 & 468 & 383 \\
\hline \multirow[t]{2}{*}{$0,10-0,15$} & 146 & 57 & 89 & 459 & 395 \\
\hline & \multicolumn{5}{|c|}{ Latossolo Vermelho distrófico típico (LVd) } \\
\hline $0,05-0,10$ & 335 & 116 & 219 & 225 & 440 \\
\hline \multirow[t]{2}{*}{$0,10-0,15$} & 338 & 112 & 226 & 210 & 452 \\
\hline & \multicolumn{5}{|c|}{ Latossolo Vermelho aluminoférrico típico (LVaf) } \\
\hline $0,05-0,10$ & 120 & 10 & 110 & 352 & 528 \\
\hline \multirow[t]{2}{*}{$0,10-0,15$} & 121 & 11 & 110 & 340 & 539 \\
\hline & \multicolumn{5}{|c|}{ Latossolo Vermelho distroférrico típico (LVdf) } \\
\hline $0,05-0,10$ & 88 & 23 & 65 & 256 & 656 \\
\hline $0,10-0,15$ & 85 & 23 & 62 & 257 & 658 \\
\hline
\end{tabular}


acordo com Silva et al. (2000), mais de $99 \%$ da compactação é alcançada nesse tempo de aplicação de carga.

Com base no deslocamento vertical, medido em laboratório, no consolidômetro, após a aplicação de cada carga, foram calculadas: a densidade do solo antes do teste de compressão (DSi) e ao final do teste (DSf), a deformação do solo ao final do teste (Def) e a umidade volumétrica antes do teste (UVi). O índice de compressão $(\mathrm{Cc})$ e a pressão de preconsolidação $(\sigma p)$ foram calculados pelo método de Casagrande (Casagrande, 1936) com ajuste manual dos dados no programa computacional Compress (Reinert et al., 2003).

Juntamente com a coleta das amostras, com estrutura de solo preservada, para o teste de compressão, foi avaliada a resistência à penetração no campo (RP), com leituras a cada $0,015 \mathrm{~m}$ de profundidade, utilizando um penetrômetro digital marca Remik CP 20 Ultrasonic Cone Penetrometer, com armazenamento eletrônico dos dados e ponta cônica com ângulo de penetração de $30^{\circ}$. A velocidade de penetração foi de aproximadamente $2 \mathrm{~m} \mathrm{~min}^{-1}$.

Considerando que a resistência à penetração é variável com a umidade e densidade do solo, utilizouse para o ajuste da RP o modelo não-linear proposto por Busscher (1990): $R P=a \theta^{b} D_{s}^{c}$, em que RP é a resistência do solo à penetração $(\mathrm{MPa}), \theta$ é a umidade volumétrica $\left(\mathrm{m}^{3} \mathrm{~m}^{-3}\right)$, Ds é a densidade do solo $\left(\mathrm{Mg} \mathrm{m}^{-3}\right)$ e $a, b, c$ são coeficientes empíricos de ajuste. Esses coeficientes do modelo não-linear foram obtidos pelo método de Gauss-Newton.

Para determinação da $\theta$, a qual será chamada de UVrp, e da Ds, chamada de DSrp, foram coletadas amostras com estrutura preservada, em cilindros com $0,030 \mathrm{~m}$ de altura e $0,055 \mathrm{~m}$ de diâmetro, nas camadas de 0,05-0,10 e 0,10-0,15 m. As amostras foram secas em estufa a $105^{\circ} \mathrm{C}$ e a densidade foi obtida conforme Blake \& Hartge (1986).

As propriedades físicas e mecânicas do solo foram submetidas à análise de correlação de Pearson e à análise de regressão linear.

\section{RESULTADOS E DISCUSSÃO}

A variação de densidade (DSrp) e umidade volumétrica (UVrp) utilizada para o ajuste da resistência à penetração, conforme equação proposta por Busscher (1990), esteve relacionada com os diferentes manejos, bem como com os diferentes teores de argila dos solos. Considerando cada solo, a DSrp apresentou uma ampla faixa de variação (Quadros 2 e 3 ).

O modelo proposto por Busscher (1990) apresentou alto ajuste com os valores de resistência do solo à penetração obtidos no campo (Figura 1), mostrando a relação da resistência à penetração com a umidade $\mathrm{e}$ densidade do solo.

Quadro 2. Médias utilizadas no ajuste do modelo (Busscher, 1990), valores máximos e mínimos, desviopadrão e coeficiente de variação da densidade (DSrp) e umidade volumétrica (UVrp), na camada de 0,05-0,15 m

\begin{tabular}{|c|c|c|c|c|c|}
\hline Variável & Média & Máximo & Mínimo & Desvio-padrão & $\mathbf{C V}^{(1)}$ \\
\hline & \multicolumn{5}{|c|}{ Argissolo Vermelho distrófico arênico (PVd2) } \\
\hline $\operatorname{DSrp}\left(\mathrm{Mg} \mathrm{m}^{-3}\right)$ & 1,70 & 1,89 & 1,36 & 0,11 & 6,69 \\
\hline \multirow[t]{2}{*}{$\operatorname{UVrp}\left(\mathrm{m}^{3} \mathrm{~m}^{-3}\right)$} & 0,21 & 0,25 & 0,18 & 0,02 & 7,81 \\
\hline & \multicolumn{5}{|c|}{ Argissolo Vermelho distrófico latossólico (PVd3) } \\
\hline $\operatorname{DSrp}\left(\mathrm{Mg} \mathrm{m}^{-3}\right)$ & 1,61 & 1,71 & 1,45 & 0,08 & 5,27 \\
\hline \multirow[t]{2}{*}{$\operatorname{UVrp}\left(\mathrm{m}^{3} \mathrm{~m}^{-3}\right)$} & 0,25 & 0,27 & 0,23 & 0,02 & 6,46 \\
\hline & \multicolumn{5}{|c|}{ Argissolo Vermelho-Amarelo alumínico típico (PVAa) } \\
\hline $\operatorname{DSrp}\left(\mathrm{Mg} \mathrm{m}^{-3}\right)$ & 1,47 & 1,52 & 1,30 & 0,06 & 4,18 \\
\hline \multirow[t]{2}{*}{$\operatorname{UVrp}\left(\mathrm{m}^{3} \mathrm{~m}^{-3}\right)$} & 0,33 & 0,37 & 0,29 & 0,02 & 5,77 \\
\hline & \multicolumn{5}{|c|}{ Latossolo Vermelho distrófico típico (LVd) } \\
\hline $\operatorname{DSrp}\left(\mathrm{Mg} \mathrm{m}^{-3}\right)$ & 1,44 & 1,59 & 1,28 & 0,11 & 7,46 \\
\hline \multirow[t]{2}{*}{$\operatorname{UVrp}\left(\mathrm{m}^{3} \mathrm{~m}^{-3}\right)$} & 0,32 & 0,37 & 0,21 & 0,04 & 12,84 \\
\hline & \multicolumn{5}{|c|}{ Latossolo Vermelho aluminoférrico típico (LVaf) } \\
\hline $\operatorname{DSrp}\left(\mathrm{Mg} \mathrm{m}^{-3}\right)$ & 1,42 & 1,59 & 1,21 & 0,10 & 7,23 \\
\hline \multirow[t]{2}{*}{$\operatorname{UVrp}\left(\mathrm{m}^{3} \mathrm{~m}^{-3}\right)$} & 0,38 & 0,42 & 0,34 & 0,02 & 5,78 \\
\hline & \multicolumn{5}{|c|}{ Latossolo Vermelho distroférrico típico (LVdf) } \\
\hline $\operatorname{DSrp}\left(\mathrm{Mg} \mathrm{m}^{-3}\right)$ & 1,39 & 1,55 & 1,04 & 0,13 & 9,46 \\
\hline $\operatorname{UVrp}\left(\mathrm{m}^{3} \mathrm{~m}^{-3}\right)$ & 0,38 & 0,42 & 0,28 & 0,04 & 11,65 \\
\hline
\end{tabular}

(1) Coeficiente de variação (\%). 
Quadro 3. Parâmetros empíricos de ajuste do modelo (Busscher, 1990) para os diferentes solos em estudo

\begin{tabular}{crrrrrr}
\hline \multirow{2}{*}{ Parâmetro } & \multicolumn{5}{c}{ Solo $^{(1)}$} \\
\cline { 2 - 6 } & PVd2 & PVd3 & PVAa & LVd & LVaf & LVdf \\
\hline & & & & & \\
a & 0,099 & 0,032 & 3,666 & 0,162 & 1,169 & 11,034 \\
b & $-0,039$ & $-1,920$ & 0,055 & $-1,075$ & $-1,008$ & 2,268 \\
c & 5,299 & 2,674 & $-1,519$ & 3,193 & $-0,971$ & 0,965 \\
\hline
\end{tabular}

(1) PVd2: Argissolo Vermelho distrófico arênico; PVd3: Argissolo Vermelho distrófico latossólico; PVAa: Argissolo VermelhoAmarelo alumínico típico; LVd: Latossolo Vermelho distrófico típico; LVaf: Latossolo Vermelho aluminoférrico típico; LVdf: Latossolo Vermelho distroférrico típico.

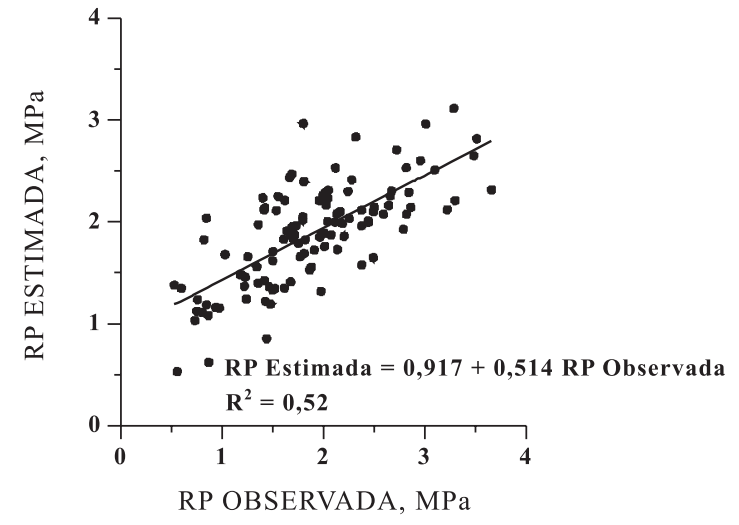

Figura 1. Relação entre a resistência do solo à penetração (RP) observada no campo e estimada a partir de dados de umidade volumétrica e densidade do solo, pela equação de Busscher (1990).

A faixa de variação da densidade (DSi) e umidade volumétrica (UVi) das amostras com estrutura preservada utilizadas no teste de compressão (Quadros 4 e 5) mostrou-se semelhante à faixa de variação da densidade (DSrp) e a umidade volumétrica (UVrp) utilizada no ajuste do modelo (Busscher, 1990) (Quadro 2).

De modo geral, com aumento do teor de argila, a densidade do solo diminuiu e a umidade volumétrica aumentou (Quadros 2, 4 e 5). A textura do solo foi influenciada pelo material de origem, sendo os solos originados de basalto mais argilosos do que os originados de material sedimentar, como o PVd2 e o PVd3. Esses últimos apresentaram constituição predominante de caulinita e quartzo na fração argila, enquanto, nos demais, verificou-se aumento da hematita (Botelho, 2005). Uma das principais características dos Latossolos é o domínio de caulinita e óxidos de $\mathrm{Fe}$ e $\mathrm{Al}$, com menores proporções de outros componentes da fração argila (Ker, 1997). Latossolos caulíniticos (Ferreira et al., 1999a,b; Centurion et al., 2007) apresentam maior densidade graças ao ajuste face a face das placas de caulinita, desenvolvendo um plasma denso (Ferreira et al., 1999a). Pedrotti et al. (2003) verificaram que o comportamento da densidade quanto a caulinita pode ser estendido para outros agrupamentos de solos. Além disso, Beutler et al. (2002) demonstraram que Latossolo caulinítico apresentou menor retenção de água do que Latossolo oxídico, embora a capacidade de água disponível tenha sido igual. Embora a mineralogia dos Latossolos em estudo tenha sido apresentada (Pombo et al., 1982; Kämpf \& Schwertmann, 1983; Dalmolin, 2002; Melo et al., 2004; Pierangeli et al., 2005; Botelho, 2005), não é possível afirmar que a maior densidade no LVd esteja relacionada com a presença de maior teor de caulinita na sua constituição, estando mais associada, portanto, à textura.

Houve correlação significativa $(p<0,01)$ e positiva entre: RP e $\sigma p$; DSi e DSf; Def e Cc; e correlação significativa ( $p<0,01)$ e negativa entre: RP e Def; DSi e Def; DSi e Cc; DSf e Def; DSf e Cc; Def e $\sigma p$ (Quadro 6). Nenhuma correlação significativa foi observada para RP e DSi, RP e DSf e RP e Cc, Dsi e $\sigma p$, DSf e $\sigma p$, Cc e $\sigma p$ (Quadro 6). Canarache et al. (2000) também observaram correlação positiva entre RP e $\sigma p$ e nenhuma correlação entre RP e Cc, porém observaram correlação entre DSi e $\sigma p$ e não observaram correlação entre DSi e Cc.

No ensaio de compressibilidade, quanto maior a densidade inicial do solo, maior a densidade final $\left(\mathrm{R}^{2}=0,57\right)$ (Figura 2a) e menor a deformação $\left(R^{2}=0,62\right)$ (Figura $\left.2 b\right)$, ou seja, quanto maior o estado de compactação, menor será a deformação do solo após a aplicação de uma pressão, pois o solo pode estar próximo de sua densidade máxima. Horn (1988) observou que, para um mesmo teor de argila, quanto menor a densidade do solo, maior sua compressibilidade. Quanto maior a densidade inicial do solo, menor sua susceptibilidade à compactação $\left(R^{2}=0,77\right)$ representada pelo índice de compressão (Figura 2c), corroborando os resultados de Silva et al. (2002). Imhoff et al. (2004) obtiveram resultados semelhantes aos deste estudo quanto à densidade, deformação e índice de compressão do solo. A menor susceptibilidade à compactação pode estar associada ao elevado estado de compactação do solo, o qual resulta em menor deformação adicional quando altas pressões (superiores à pressão de preconsolidação) são aplicadas ao solo. 
Quadro 4. Média, máximo, mínimo, desvio-padrão e coeficiente de variação da resistência do solo à penetração (RP); umidade volumétrica inicial (UVi), densidade do solo antes e ao final do teste de compressão (DSi e DSf), deformação do solo (Def), índice de compressão (Cc) e pressão de preconsolidação ( $\sigma \mathrm{p})$, para os Argissolos em estudo

\begin{tabular}{|c|c|c|c|c|c|}
\hline Variável & Média & Máximo & Mínimo & Desvio-padrão & $\mathrm{CV}^{(1)}$ \\
\hline \multicolumn{6}{|c|}{ Argissolo Vermelho distrófico arênico (PVd2) } \\
\hline $\mathrm{RP}(\mathrm{MPa})$ & 1,86 & 3,11 & 0,53 & 0,61 & 32,87 \\
\hline UVi $\left(\mathrm{m}^{3} \mathrm{~m}^{-3}\right)$ & 0,15 & 0,24 & 0,10 & 0,02 & 15,47 \\
\hline $\mathrm{DSi}\left(\mathrm{Mg} \mathrm{m}^{-3}\right)$ & 1,61 & 1,81 & 1,41 & 0,09 & 5,73 \\
\hline $\mathrm{DSf}\left(\mathrm{Mg} \mathrm{m}^{-3}\right)$ & 1,86 & 1,99 & 1,77 & 0,04 & 2,46 \\
\hline Def $(\mathrm{mm})$ & 0,34 & 0,57 & 0,18 & 0,09 & 27,38 \\
\hline $\mathrm{Cc}$ & 0,18 & 0,34 & 0,07 & 0,06 & 35,29 \\
\hline$\sigma \mathrm{p}(\mathrm{kPa})$ & 134,1 & 195,9 & 63,7 & 35,7 & 26,5 \\
\hline \multicolumn{6}{|c|}{ Argissolo Vermelho distrófico latossólico (PVd3) } \\
\hline $\mathrm{RP}(\mathrm{MPa})$ & 1,65 & 2,14 & 1,15 & 0,34 & 21,09 \\
\hline UVi $\left(\mathrm{m}^{3} \mathrm{~m}^{-3}\right)$ & 0,22 & 0,26 & 0,18 & 0,02 & 10,00 \\
\hline $\mathrm{DSi}\left(\mathrm{Mg} \mathrm{m}^{-3}\right)$ & 1,60 & 1,69 & 1,48 & 0,06 & 3,84 \\
\hline $\mathrm{DSf}\left(\mathrm{Mg} \mathrm{m}^{-3}\right)$ & 1,90 & 1,93 & 1,84 & 0,03 & 1,40 \\
\hline $\operatorname{Def}(\mathrm{mm})$ & 0,40 & 0,54 & 0,28 & 0,07 & 18,30 \\
\hline $\mathrm{Cc}$ & 0,20 & 0,27 & 0,13 & 0,04 & 21,28 \\
\hline$\sigma \mathrm{p}(\mathrm{kPa})$ & 122,8 & 176,5 & 78,2 & 35,3 & 28,7 \\
\hline \multicolumn{6}{|c|}{ Argissolo Vermelho-Amarelo alumínico típico (PVAa) } \\
\hline $\mathrm{RP}(\mathrm{MPa})$ & 1,92 & 2,30 & 1,83 & 0,13 & 6,81 \\
\hline UVi $\left(\mathrm{m}^{3} \mathrm{~m}^{-3}\right)$ & 0,32 & 0,37 & 0,26 & 0,03 & 9,77 \\
\hline DSi $\left(\mathrm{Mg} \mathrm{m}^{-3}\right)$ & 1,50 & 1,58 & 1,40 & 0,06 & 4,19 \\
\hline $\operatorname{DSf}\left(\mathrm{Mg} \mathrm{m}^{-3}\right)$ & 1,79 & 1,84 & 1,72 & 0,04 & 2,32 \\
\hline Def $(\mathrm{mm})$ & 0,36 & 0,52 & 0,26 & 0,07 & 18,82 \\
\hline $\mathrm{Cc}$ & 0,23 & 0,34 & 0,15 & 0,05 & 24,55 \\
\hline$\sigma \mathrm{p}(\mathrm{kPa})$ & 124,8 & 165,4 & 95,4 & 22,9 & 18,3 \\
\hline
\end{tabular}

${ }^{(1)}$ Coeficiente de variação (\%).

Quadro 5. Média, máximo, mínimo, desvio-padrão e coeficiente de variação da resistência do solo à penetração (RP); umidade volumétrica (UVi), densidade do solo antes e ao final do teste de compressão (DSi e DSf), deformação do solo (Def), índice de compressão (Cc) e pressão de preconsolidação ( $\sigma p)$, para os Latossolos em estudo

\begin{tabular}{|c|c|c|c|c|c|}
\hline Variável & Média & Máximo & Mínimo & Desvio-padrão & $\mathbf{C V}^{(1)}$ \\
\hline \multicolumn{6}{|c|}{ Latossolo Vermelho distrófico típico (LVd) } \\
\hline $\mathrm{RP}(\mathrm{MPa})$ & 1,79 & 2,29 & 1,34 & 0,35 & 19,70 \\
\hline $\operatorname{UVi}\left(\mathrm{m}^{3} \mathrm{~m}^{-3}\right)$ & 0,31 & 0,35 & 0,27 & 0,02 & 8,10 \\
\hline $\mathrm{DSi}\left(\mathrm{Mg} \mathrm{m}^{-3}\right)$ & 1,48 & 1,63 & 1,33 & 0,09 & 6,08 \\
\hline $\operatorname{DSf}\left(\mathrm{Mg} \mathrm{m}^{-3}\right)$ & 1,77 & 1,82 & 1,70 & 0,04 & 2,55 \\
\hline Def $(\mathrm{m} \mathrm{m})$ & 0,41 & 0,55 & 0,25 & 0,08 & 20,63 \\
\hline $\mathrm{Cc}$ & 0,24 & 0,35 & 0,12 & 0,06 & 27,41 \\
\hline$\sigma \mathrm{p}(\mathrm{kPa})$ & 118,7 & 161,0 & 95,6 & 20,9 & 17,6 \\
\hline \multicolumn{6}{|c|}{ Latossolo Vermelho aluminoférrico típico (LVaf) } \\
\hline $\mathrm{RP}(\mathrm{MPa})$ & 2,23 & 2,71 & 1,96 & 0,22 & 9,96 \\
\hline $\operatorname{UVi}\left(\mathrm{m}^{3} \mathrm{~m}^{-3}\right)$ & 0,36 & 0,43 & 0,27 & 0,05 & 14,35 \\
\hline $\mathrm{DSi}\left(\mathrm{Mg} \mathrm{m}^{-3}\right)$ & 1,43 & 1,58 & 1,28 & 0,08 & 5,72 \\
\hline $\operatorname{DSf}\left(\mathrm{Mg} \mathrm{m}^{-3}\right)$ & 1,76 & 1,93 & 1,60 & 0,08 & 4,50 \\
\hline Def $(m \mathrm{~m})$ & 0,42 & 0,65 & 0,31 & 0,08 & 19,48 \\
\hline $\mathrm{Cc}$ & 0,31 & 0,45 & 0,18 & 0,07 & 22,18 \\
\hline$\sigma \mathrm{p}(\mathrm{kPa})$ & 128,8 & 160,4 & 99,1 & 19,7 & 15,3 \\
\hline \multicolumn{6}{|c|}{ Latossolo Vermelho distroférrico típico (LVdf) } \\
\hline $\mathrm{RP}(\mathrm{MPa})$ & 1,76 & 2,21 & 0,62 & 0,51 & 29,08 \\
\hline UVi $\left(\mathrm{m}^{3} \mathrm{~m}^{-3}\right)$ & 0,36 & 0,41 & 0,30 & 0,04 & 11,46 \\
\hline $\mathrm{DSi}\left(\mathrm{Mg} \mathrm{m}^{-3}\right)$ & 1,40 & 1,55 & 1,15 & 0,13 & 9,25 \\
\hline $\operatorname{DSf}\left(\mathrm{Mg} \mathrm{m}^{-3}\right)$ & 1,68 & 1,70 & 1,64 & 0,02 & 1,19 \\
\hline $\operatorname{Def}(\mathrm{m} \mathrm{m})$ & 0,41 & 0,80 & 0,20 & 0,20 & 48,24 \\
\hline $\mathrm{Cc}$ & 0,22 & 0,38 & 0,12 & 0,10 & 42,93 \\
\hline$\sigma \mathrm{p}(\mathrm{kPa})$ & 132,7 & 185,7 & 54,6 & 39,2 & 29,6 \\
\hline
\end{tabular}

(1) Coeficiente de variação (\%). 
Quadro 6. Matriz de correlação de Pearson das variáveis físicas e mecânicas do solo

\begin{tabular}{lcclll}
\hline & RP & DSi & DSf & Def & Cc \\
\hline DSi & $0,16^{\text {ns }}$ & - & - & - & - \\
DSf & $-0,01^{\text {ns }}$ & $0,76^{* *}$ & - & - & - \\
Def & $-0,46^{* *}$ & $-0,79^{* *}$ & $-0,30^{* *}$ & - & - \\
Cc & $-0,03^{\text {ns }}$ & $-0,88^{* *}$ & $-0,43^{* *}$ & $0,83^{* *}$ & - \\
$\sigma p$ & $0,68^{* *}$ & $0,20^{\text {ns }}$ & $-0,01^{\text {ns }}$ & $-0,37^{* *}$ & $0,05^{\text {ns }}$ \\
\hline
\end{tabular}

RP: resistência do solo à penetração ajustada pela densidade e umidade; DSi: densidade do solo antes do teste de compressão; DSf: densidade do solo ao final do teste de compressão; Def: deformação do solo ao final do teste de compressão; Cc: índice de compressão, $\sigma$ p: pressão de preconsolidação. ${ }^{* *}$ : significativo a $1 \%$ e ns: não-significativo.

Embora a densidade não tenha apresentado relação com a pressão de preconsolidação (Figura 3d), trabalhos têm indicado que, quanto maior a densidade, maior a pressão de preconsolidação (Canarache et al., 2000; Imhoff et al., 2001, 2004). Alexandrou \& Earl (1998) observaram relação significativa entre pressão de preconsolidação e densidade inicial para solo arenoso, mas nenhuma relação foi observada para um solo argiloso, fato atribuído à dominância de coesão e menor fricção no solo argiloso. Mosaddeghi et al. (2003) não observaram correlação entre pressão de preconsolidação e densidade, porém observaram aumento da pressão de preconsolidação com aumento da densidade do solo. Segundo Lebert \& Horn (1991), a influência da densidade na pressão de preconsolidação é reduzida com aumento do teor de argila.

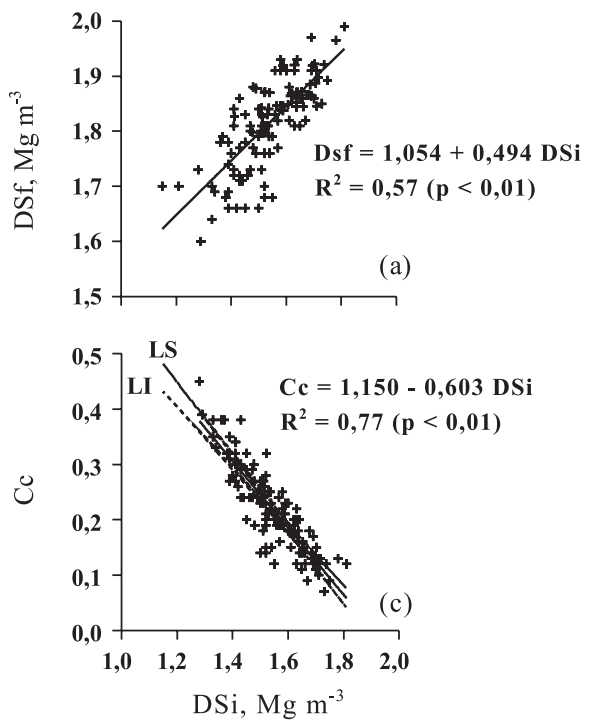

A resistência à penetração não apresentou relação com a densidade final (Figura 3a) e com o índice de compressão (Figura 3c). Com o aumento da resistência à penetração, ocorreu uma redução da deformação do solo $\left(\mathrm{R}^{2}=0,20\right)$ (Figura $\left.3 \mathrm{~b}\right)$ e aumento da pressão de preconsolidação $\left(\mathrm{R}^{2}=0,46\right)$ (Figura $\left.3 \mathrm{~d}\right)$; assim, o solo com maior resistência à penetração suporta maiores pressões. Porém, deve ser observado até que ponto o solo poderia suportar tal pressão sem prejuízo à sua estrutura e ao crescimento e desenvolvimento das plantas.

Canarache et al. (2000) e Dias Júnior et al. (2004) obtiveram relação linear entre resistência à penetração e pressão de preconsolidação, corroborando o obtido neste trabalho. A relação entre $\sigma p$ e RP observada no estudo foi de 15:1, enquanto Canarache et al. (2000), para um solo com aproximadamente $228 \mathrm{~g} \mathrm{~kg}^{-1}$ de argila, e Mosaddeghi et al. (2003), para um solo com $348 \mathrm{~g} \mathrm{~kg}^{-1}$ de argila, verificaram uma relação de 10:1, e Lima et al. (2006), para um Latossolo Vermelho Amarelo distrófico com $170 \mathrm{~g} \mathrm{~kg}^{-1}$ de argila, sob pomar de laranja, verificaram uma relação de 17:1. A menor relação obtida por Canarache et al. (2000) e Mosaddeghi et al. (2003) pode estar associada ao fato de eles terem trabalhado com solo revolvido, ou seja, com estrutura perturbada. Considerando a relação op e RP, para uma resistência à penetração de $2 \mathrm{MPa}$, a pressão de preconsolidação correspondente seria de $200 \mathrm{kPa}$ para a relação 10:1, $133 \mathrm{kPa}$ para a relação 15:1 e $118 \mathrm{kPa}$ para a relação 17:1. Essas relações permitem que a avaliação da resistência à penetração, que é prática e simples, seja usada para estimar a pressão de preconsolidação, que exige equipamento específico e maior tempo para obtenção dos dados. Tomando a equação da figura 3d, observa-se que o
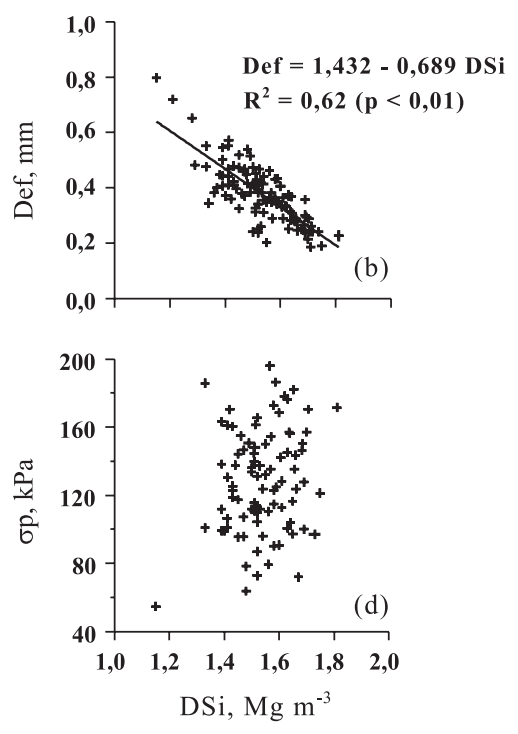

Figura 2. Relações entre densidade do solo antes de teste de compressão (DSi) e densidade ao final do teste (DSf) (a), deformação ao final do teste (Def) (b), índice de compressão (Cc) (c) e pressão de preconsolidação $(\sigma p)(d)$. LS: limite superior do intervalo de confiança; LI: limite inferior do intervalo de confiança. 

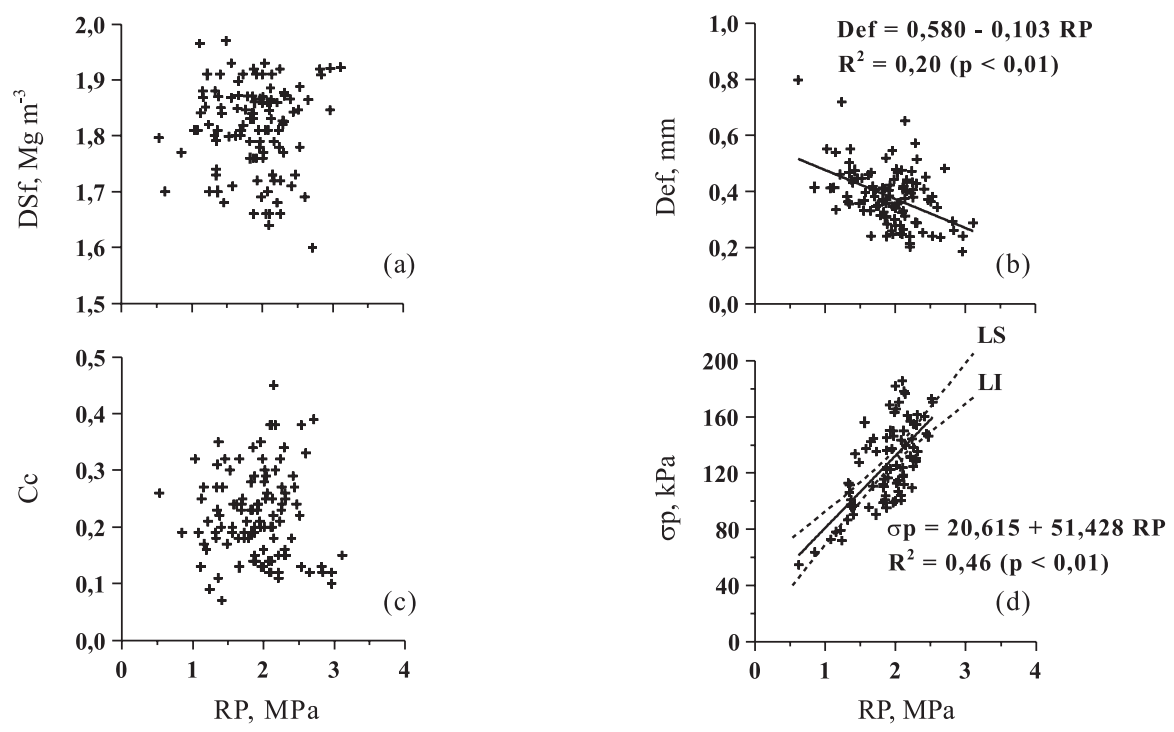

Figura 3. Relações entre resistência do solo à penetração (RP) e densidade ao final do teste de compressão (DSf) (a), deformação ao final do teste (Def) (b), índice de compressão (Cc) (c) e pressão de preconsolidação $(\sigma p)(d)$. LS: limite superior do intervalo de confiança; LI: limite inferior do intervalo de confiança.

valor de $\sigma p$, quando $\mathrm{RP}=2 \mathrm{MPa}$, é equivalente a $133 \mathrm{kPa}$, variando de 127 a $137 \mathrm{kPa}$ (intervalo de confiança de $95 \%$ ).

A deformação do solo relacionou-se com o índice de compressão $\left(\mathrm{R}^{2}=0,69\right)$ (Figura $\left.4 \mathrm{a}\right)$ e com a pressão de preconsolidação $\left(\mathrm{R}^{2}=0,12\right)$ (Figura $\left.4 \mathrm{~b}\right)$. Quanto maior a deformação de um solo, maior sua susceptibilidade à compactação e menor sua capacidade de suporte de carga, embora, para este último caso, o coeficiente de determinação, que expressa quanto da variação da pressão de preconsolidação é explicada pela deformação do solo, tenha sido baixo $\left(\mathrm{R}^{2}=0,12\right)$.

Nas figuras 5a,b, foram utilizados os valores observados deíndice de compressão, pressão de preconsolidação e teor de argila (camada de 0,05-0,15 m) apenas das áreas há mais tempo sob plantio direto.

O índice de compressão $\left(\mathrm{R}^{2}=0,70\right)$ e de pressão de preconsolidação $\left(\mathrm{R}^{2}=0,32\right)$ mostrou-se dependente do teor de argila do solo (Figura 5a,b). Embora uma função linear tenha melhor representado a relação entre o índice de compressão e o teor de argila, notase que o índice de compressão tende a aumentar até um valor de argila de aproximadamente $390 \mathrm{~g} \mathrm{~kg}^{-1}$, permanecendo relativamente constante daí para frente (Figura 5a). Essa tendência poderia ser confirmada com maior número de observações. Outros trabalhos também têm apresentado relação positiva entre teor de argila e índice de compressão (Arvidsson, 1998). Larson et al. (1980), trabalhando com amostras de solo com estrutura alterada, e Imhoff et al. (2004), trabalhando com amostras com estrutura preservada, observaram aumento do índice de compressão até um valor de argila de aproximadamente 330 e $300 \mathrm{~g} \mathrm{~kg}^{-1}$, respectivamente. Smith et al. (1997a,b) também observaram que solos arenosos são menos compressivos
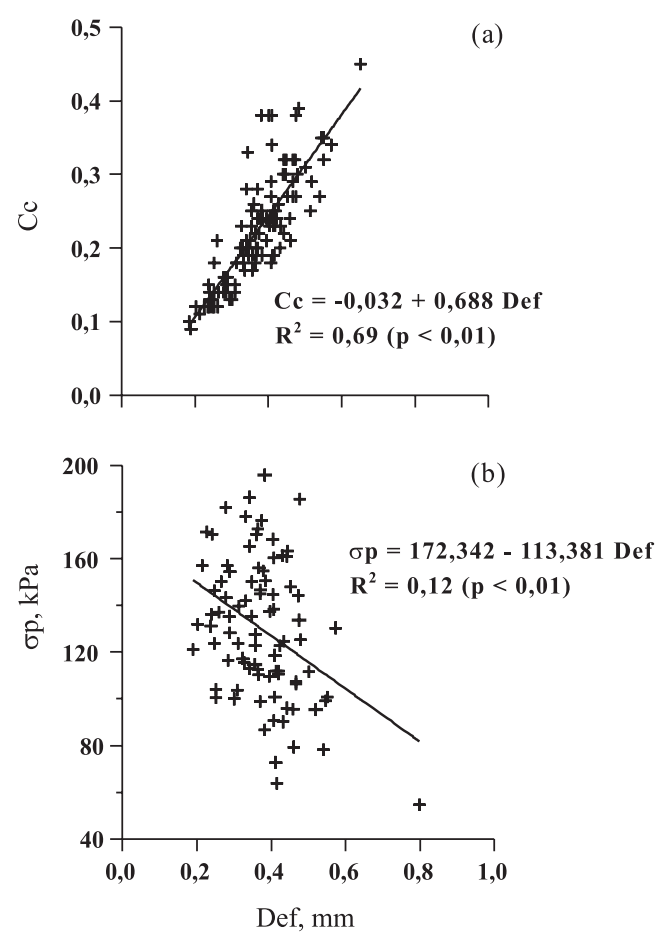

Figura 4. Relação entre a deformação do solo ao final do teste de compressão (Def) e índice de compressão (Cc) (a) e pressão de preconsolidação $(\sigma p)(b)$.

que solos argilosos. Esse aumento do índice de compressão até aproximadamente $390 \mathrm{~g} \mathrm{~kg}^{-1}$ de argila pode estar associado ao decréscimo do teor de caulinita e ao incremento de óxidos com aumento do teor de argila desses solos em estudo. 

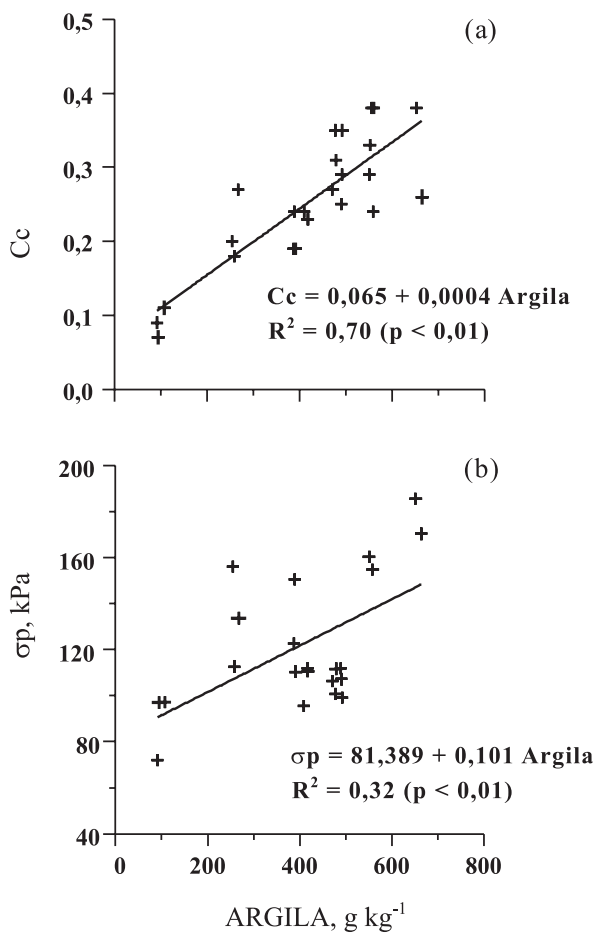

Figura 5. Comportamento do índice de compressão (Cc) (a) e pressão de preconsolidação $\sigma p$ (b) de acordo com o teor de argila.

Um aumento na capacidade de suporte de carga do solo ocorreu com aumento do teor de argila (Figura 5b), fato também verificado por Imhoff et al. (2004). Solos argilosos são capazes de suportar maiores pressões (Figura 5b), porém são mais susceptíveis à compactação (Figura 5a), fato que pode estar associado à capacidade de os solos argilosos manterem maior umidade a maiores tensões, e a umidade está relacionada com a susceptibilidade do solo à compactação (Silva et al., 2002). Beutler et al. (2002) verificaram que Latossolo caulinítico apresentou menor retenção de água do que Latossolo oxídico, embora a capacidade de água disponível tenha sido igual.

O solo apresenta limites no suporte de pressões. Portanto, é necessário verificar quais são esses valores para que se possa mapear ou classificar os solos quanto à sua capacidade de suporte e susceptibilidade à compactação, para um adequado manejo do solo e crescimento e desenvolvimento das plantas.

De acordo com os resultados obtidos, a susceptibilidade à compactação, representada pelo índice de compressão, pode ser estimada pela densidade inicial, enquanto a capacidade de suporte de carga do solo, representada pela pressão de preconsolidação, pode ser estimada pela resistência à penetração. Deve-se observar que esses resultados foram obtidos para condições específicas de umidade e com avaliações na camada de maior compactação $(0,05-0,15 \mathrm{~m})$, em sistema plantio direto, ocasionado pelas pressões exercidas por máquinas e equipamentos, quando o solo estava com umidade excessiva. Considerando que a concentração das pressões em sistema plantio direto ocorre nessa camada de estudo, é possível que esses resultados possam ser extrapolados para outras camadas ou, pelo menos, possam auxiliar na tomada de decisões relacionadas com o manejo do solo para evitar ou minimizar os efeitos negativos do tráfego de máquinas ao solo.

\section{CONCLUSÕES}

1. Os valores de resistência à penetração ajustados pelo modelo que considera a umidade e densidade do solo apresentaram relação significativa com os valores obtidos em campo.

2. Solos sob plantio direto com maior densidade inicial na camada de 0,05-0,15 m apresentaram menor susceptibilidade à compactação e menor deformação, quando submetidos a pressões externas.

3. Quanto maior a resistência do solo à penetração, menor sua deformação e maior capacidade de suporte de carga, embora isso não indique solos com qualidade física adequada para as culturas.

4. Quanto maior a deformação total da amostra de solo no teste de compressão uniaxial, mais susceptível à compactação e menor sua capacidade de suporte de carga.

5. A susceptibilidade do solo à compactação e sua capacidade de suporte de carga podem ser estimadas, respectivamente, pela densidade inicial e pela resistência à penetração.

\section{AGRADECIMENTOS}

Aos bolsistas de iniciação científica do Setor de Física do Solo da Universidade Federal de Santa Maria, pelo auxílio nos trabalhos de campo e laboratório. À Capes, ao CNPq e à Fapergs, pelas bolsas de pesquisa.

\section{LITERATURA CITADA}

ALEXANDROU, A. \& EARL, R. The relationship among the pre-compaction stress, volumetric water content and initial dry bulk density of soil. J. Agric. Eng. Res., 71:7580, 1998.

ARVIDSSON, J. Influence of soil texture and organic matter content on bulk density, air content, compression index and crop yield in field and laboratory compression experiments. Soil Till. Res., 49:159-170, 1998.

BEUTLER, A.N.; CENTURION, J.F.; SOUZA, Z.M.; ANDRIOLI, I. \& ROQUE, C.G. Retenção de água em dois tipos de Latossolos sob diferentes usos. R. Bras. Ci. Solo, 26:829-834, 2002. 
BLAKE, G.R. \& HARTGE, K.H. Bulk density. In: KLUTE, A. Methods of soil analysis: Physical and mineralogical methods. 2.ed. Madison, American Society of Agronomy, Soil Science Society of America, 1986. p.363-375.

BOTELHO, M.R. Avaliação da cor e comportamento espectral de algumas classes de solos do Rio Grande do Sul. Santa Maria, Universidade Federal de Santa Maria, 2005. 103p. (Tese de Mestrado)

BRASIL. Ministério da Agricultura. Departamento Nacional de Pesquisa Agropecuária. Divisão de Pesquisa Pedológica. Levantamento de reconhecimento dos solos do estado do Rio Grande do Sul. Recife, 1973. (Boletim Técnico, 30)

BUSSCHER, W.J. Adjustment of flat-tipped penetrometer resistance data to a commom water content. Trans. Am. Soc. Agric. Eng., 3:519-524, 1990.

CANARACHE, A.; HORN, R. \& COLIBAS, I. Compressibility of soils in a long term field experiment with intensive deep ripping in Romania. Soil Till. Res., 56:185-196, 2000.

CASAGRANDE, A. The determination of the pre-consolidation load and its practical significance. In: INTERNATIONAL CONFERENCE ON SOIL MECHANICS AND FOUNDATION ENGINEERING, 1936, Cambridge. Proceedings. Cambridge, Harvard University, 1936. p.6064.

CENTURION, J.F.; FREDDI, O.S.; ARATANI, R.G.; METZNER, A.F.M.; BEUTLER, A.N. \& ANDRIOLI, I. Influência do cultivo da cana-de-açúcar e da mineralogia da fração argila nas propriedades físicas de Latossolos Vermelhos. R. Bras. Ci. Solo, 31:199-209, 2007.

DALMOLIN, R.S.D. Matéria orgânica e características físicas, químicas e mineralógicas e espectrais de Latossolos de diferentes ambientes. Porto Alegre, Universidade Federal do Rio Grande do Sul, 2002. 152p. (Tese de Doutorado)

DIAS JÚNIOR, M.S.; SILVA, A.R.; FONSECA, S. \& LEITE, F.P. Método alternativo de avaliação da pressão de preconsolidação por meio de um penetrômetro. R. Bras. Ci. Solo, 28:805-810, 2004.

EMPRESA BRASILEIRA DE PESQUISA AGROPECUÁRIA EMBRAPA. Centro Nacional de Pesquisa de Solos. Manual de métodos de análise de solos. 2.ed. Rio de Janeiro, 1997. 212p.

FERREIRA, M.M.; FERNANDES, B. \& CURI, N. Influência da mineralogia da fração argila nas propriedades físicas de Latossolos da região sudeste do Brasil. R. Bras. Ci. Solo, 23:515-524, 1999b.

FERREIRA, M.M.; FERNANDES, B. \& CURI, N. Mineralogia da fração argila e estrutura de Latossolos da região sudeste do Brasil. R. Bras. Ci. Solo, 23:507-514, 1999a.

GENRO JUNIOR, S.A.; REINERT, D.J. \& REICHERT, J.M. Variabilidade temporal da resistência à penetração de um Latossolo argiloso sob semeadura direta com rotação de culturas. R. Bras. Ci. Solo, 28:477-484, 2004.

HORN, R. Compressibility of arable land. Catena Suppl., 11:53$71,1988$.

HORN, R. \& FLEIGE, H. A method for assessing the impact of load on mechanical stability and on physical properties of soils. Soil Till. Res., 73:89-99, 2003.
IMHOFF, S.; SILVA, A.P. \& FALLOW, D. Susceptibility to compaction, load support capacity, and soil compressibility of Hapludox. Soil Sci. Soc. Am. J., 68:17-24, 2004.

IMHOFF, S.; SILVA, A.P.; DIAS JÚNIOR, M.S. \& TORMENA, C.A. Quantificação de pressões críticas para o crescimento das plantas. R. Bras. Ci. Solo, 25:11-18, 2001.

KÄMPF, N. \& SCHWERTMANN, U. Relações entre óxidos de ferro e a cor em solos cauliníticos do Rio Grande do Sul. R. Bras. Ci. Solo, 7:27-31, 1983.

KER, J.C. Latossolos do Brasil: Uma revisão. Geonomos, 5:1740, 1997.

KLUTE, A. Water retention: Laboratory methods. In: KLUTE, A., ed. Methods of soil analysis: Physical and mineralogical methods. 2. ed. Madison, American Society of Agronomy, Soil Science Society of America, 1986. p.635-660.

LARSON, W.E.; GUPTA, S.C. \& USECHE, R.A. Compression of agricultural soils from eight soil orders. Soil Sci. Soc. Am. J., 44:450-457, 1980.

LEBERT, M. \& HORN, R. A method to predict the mechanical strength of agricultural soils. Soil Till. Res., 19:275-286, 1991.

LIMA, C.L.R.; SILVA, A.P.; IMHOFF, S. \& LEÃO, T.P. Estimativa da capacidade de suporte de carga do solo a partir da avaliação da resistência à penetração. R. Bras. Ci. Solo, 30:217-223, 2006.

MELO, G.W.; MEURER, E.J. \& PINTO, L.F.S. Fontes de potássio em solos distroférricos cauliníticos originados de basalto no Rio Grande do Sul. R. Bras. Ci. Solo, 28:597603, 2004

MOSADDEGHI, M.R.; HEMMAT, A.; HAJABBASI, M.A. \& ALEXANDROU, A. Pre-compression stress and its relation with the physical and mechanical properties of a structurally unstable soil in central Iran. Soil Till. Res., 70:53-64, 2003

PEDROTTI, A.; FERREIRA, M.M.; CURI, N.; SILVA, M.L.N.; LIMA, J.M. \& CARVALHO, R. Relação entre atributos físicos, mineralogia da fração argila e formas de alumínio no solo. R. Bras. Ci. Solo, 27:1-9, 2003.

PIERANGELI, M.A.P.; GUILHERME, L.R.G.; CURI, N.; SILVA, M.L.N.; LIMA, J.M. \& COSTA, E.T.S. Efeito do pH na adsorção e dessorção de cádmio em Latossolos brasileiros. R. Bras. Ci. Solo, 29:523-532, 2005.

POMBO, L.C.A.; KLAMT, E.; KUNRATH, I. \& GIANLUPPI, D. Identificação de óxidos de ferro na fração argila de Latossolo Roxo. R. Bras. Ci. Solo, 6:13-17, 1982.

REINERT, D.J.; ROBAINA, A. \& REICHERT, J.M. Compress - software e proposta de modelo para descrever a compressibilidade dos solos e seus parâmetros. In: CONGRESSO BRASILEIRO DE CIÊNCIA DO SOLO, 29., 2003, Ribeirão Preto. Anais. Ribeirão Preto, Sociedade Brasileira de Ciência do Solo, 2003. CD-ROM.

SANTOS, H.G.; JACOMINE, P.K.T.; ANJOS, L.H.; OLIVEIRA, V.A.; OLIVEIRA, J.B.; COELHO, M.R.; LUMBRERAS, J.F. \& CUNHA, T.J.F., eds. Sistema brasileiro de classificação de solos. 2.ed. Rio de Janeiro, Embrapa Solos, 2006. 306p. 
SILVA, V.R. Propriedades físicas e hídricas em solos sob diferentes estados de compactação. Santa Maria, Universidade Federal de Santa Maria, 2003. 171p. (Tese de Doutorado)

SILVA, V.R.; REINERT, D.J. \& REICHERT, J.M. Fatores controladores da compressibilidade de um Argissolo Vermelho-Amarelo distrófico arênico e de um Latossolo Vermelho distrófico típico. II - Grau de saturação em água. R. Bras. Ci. Solo, 26:9-15, 2002.

SILVA, V.R.; REINERT, D.J. \& REICHERT, J.M. Susceptibilidade à compactação de um Latossolo Vermelho-Escuro e de um Podzólico Vermelho-Amarelo. R. Bras. Ci. Solo, 4:239-249, 2000.

SMITH, C.W.; JOHNSTON, M.A. \& LORENTZ, S. Assessing the compaction susceptibility of South African forestry soils. I. The effect of soil type, water content and applied pressure on uni-axial compaction. Soil Till. Res., 41:5373, 1997a.

SMITH, C.W.; JOHNSTON, M.A. \& LORENTZ, S. Assessing the compaction susceptibility of South African forestry soils. II. Soil properties affecting compactability and compressibility. Soil Till. Res., 43:335-354, $1997 \mathrm{~b}$.
STRECK, E.V.; KÄMPF, N.; DALMOLIN, R.S.D.; KLAMT, E.; NASCIMENTO, P.C. \& SCHNEIDER, P. Solos do Rio Grande do Sul. Porto Alegre, Emater/RS; UFRGS, 2002. 126 p.

SUZUKI, L.E.A.S. Compactação do solo e sua influência nas propriedades físicas. Santa Maria, Universidade Federal de Santa Maria, 2005. 149p. (Tese de Mestrado)

SUZUKI, L.E.A.S.; REINERT, D.J.; KAISER, D.R.; KUNZ, M.; PELLEGRINI, A.; REICHERT, J.M. \& ALBUQUERQUE, J.A. Teor de argila de solos sob diferentes tempos de agitação horizontal, tempo de contato do dispersante químico e dispersão mecânica. In: REUNIÃO BRASILEIRA DE MANEJO E CONSERVAÇÃO DO SOLO E DA ÁGUA, 15., 2004, Santa Maria. Anais. Santa Maria, Sociedade Brasileira de Ciência do Solo, 2004a. CD-ROM.

SUZUKI, L.E.A.S.; REINERT, D.J.; KAISER, D.R.; KUNZ, M.; PELLEGRINI, A.; REICHERT, J.M. \& ALBUQUERQUE, J.A. Areia total de solos sob diferentes tempos de agitação horizontal, tempo de contato do dispersante químico e dispersão mecânica. In: REUNIÃO BRASILEIRA DE MANEJO E CONSERVAÇÃO DO SOLO E DA ÁGUA, 15., 2004, Santa Maria. Anais. Santa Maria, Sociedade Brasileira de Ciência do Solo, 2004b. CD-ROM. 